\title{
Systematic uncertainties on the cosmic-ray transport parameters
}

\section{Is it possible to reconcile $\mathrm{B} / \mathrm{C}$ data with $\delta=1 / 3$ or $\delta=1 / 2$ ?}

\author{
D. Maurin ${ }^{1,2,3}$, A. Putze ${ }^{4,5}$, and L. Derome ${ }^{4}$ \\ ${ }^{1}$ Laboratoire de Physique Nucléaire et des Hautes Énergies (LPNHE), Universités Paris VI et Paris VII, CNRS/IN2P3, Tour 33, \\ Jussieu, Paris, 75005, France \\ e-mail: dmaurin@lpnhe.in2p3.fr \\ 2 Dept. of Physics and Astronomy, University of Leicester, Leicester, LE17RH, UK \\ 3 Institut d'Astrophysique de Paris (IAP), UMR 7095 CNRS, Université Pierre et Marie Curie, 98bis Bd Arago, 75014 Paris, France \\ ${ }^{4}$ Laboratoire de Physique Subatomique et de Cosmologie (LPSC), Université Joseph Fourier Grenoble 1, CNRS/IN2P3, \\ Institut Polytechnique de Grenoble, 53 avenue des Martyrs, 38026 Grenoble, France \\ 5 The Oskar Klein Centre for Cosmoparticle Physics, Department of Physics, Stockholm University, AlbaNova, 10691 Stockholm, \\ Sweden
}

Received 7 January 2010 / Accepted 14 March 2010

\section{ABSTRACT}

\begin{abstract}
Context. The $\mathrm{B} / \mathrm{C}$ ratio is used in cosmic-ray physics to constrain the transport parameters. However, from the same set of data, the various published values show a puzzling large scatter of these parameters.

Aims. We investigate the impact of using different inputs (gas density and hydrogen fraction in the Galactic disc, source spectral shape, low-energy dependence of the diffusion coefficient, and nuclear fragmentation cross-sections) on the best-fit values of the transport parameters. We quantify the systematics produced when varying these inputs, and compare them to statistical uncertainties. We discuss the consequences for the slope of the diffusion coefficient $\delta$.

Methods. The analysis relies on the propagation code USINE interfaced with the Minuit minimisation routines.

Results. We find the typical systematic uncertainties to be greater than the statistical ones. The several published values of $\delta$ (from 0.3 to 0.8 ) can be recovered when varying the low-energy shape of the diffusion coefficient and the convective wind strength. Models including a convective wind are characterised by $\delta \gtrsim 0.6$, which cannot be reconciled with the expected theoretical values $(1 / 3$ and $1 / 2)$. However, from a statistical point of view ( $\chi^{2}$ analysis), models with both reacceleration and convection - hence large $\delta-$ are favoured. The next favoured models in line yield $\delta$, which can be accommodated with $1 / 3$ and $1 / 2$, but require a strong upturn of the diffusion coefficient at low energy (and no convection).

Conclusions. To date, using the best statistical tools, the transport parameter determination is still plagued by many unknowns at low energy $(\sim \mathrm{GeV} / \mathrm{n})$. To disentangle all these configurations, measurements of the $\mathrm{B} / \mathrm{C}$ ratio at $\mathrm{TeV} / \mathrm{n}$ energies and/or combination with other secondary-to-primary ratios is necessary.
\end{abstract}

Key words. methods: statistical - cosmic rays

\section{Introduction}

A central question in Galactic cosmic ray (GCR) physics lies in the determination of the transport parameters. The standard procedure consists in fitting a secondary-to-primary ratio, e.g. B/C. In general, the parameters derived in different studies provide inconsistent values, especially for the slope $\delta$ of the diffusion coefficient. It is important to understand the origin of these differences.

The first attempts to get the best-fit value of the transport parameters, as well as their statistical uncertainties, were carried in Maurin et al. (2001) and Lionetto et al. (2005). Recently, we implemented a Markov chain Monte Carlo (MCMC) to estimate the probability-density function (PDF) of the transport and source parameters for Galactic cosmic rays (Putze et al. 2009, 2010, hereafter Papers I and II). This sound statistical technique applied to current $\mathrm{B} / \mathrm{C}$ data allowed us to estimate the statistical uncertainties on various parameters. We found typical uncertainties of $\sim 10-20 \%$. Although such precision is expected given the accuracy of the data, one may wonder whether these results are not dominated by systematic uncertainties from the input ingredients and assumptions made to do the calculation.

This is an important issue since in our previous studies, the best-fit slope observed in a diffusion/constantconvection/reacceleration propagation model (Maurin et al. 2001, 2002) - confirmed by our recent MCMC analysis (Paper II) - points to $\delta \approx 0.75-0.85$. This is at variance with the result of the GALPROP code (Strong \& Moskalenko 1998), where the best-fit models correspond to smaller $\delta \approx 0.3-0.4$ (Lionetto et al. 2005) in either a diffusion/linear-convection or a diffusion - reacceleration model. Moreover, none of these results agree with the best-fit slopes from leaky-box models (with rigidity cut-off), where $\delta \approx 0.5-0.6$ (e.g., Webber et al. $2003-$ no reacceleration - , or Paper I - with reacceleration). Such a scatter in $\delta$ was also observed in Jones et al. (2001).

The propagation code USINE (Maurin et al., in prep.) provides the GCR fluxes in both the framework of the leaky-box model (LBM) and the diffusion model (DM). Associated with an efficient minimisation tool for finding the best-fit parameters of a model (w/wo convection, w/wo diffusive reacceleration), it allows these differences to be addressed thoroughly. We also 
investigate how sensitive these best-fit parameters are to various input ingredients/parameters.

The paper is organised as follows. In Sect. 2, the DM used is briefly recalled. In Sect. 3, the free parameters of the study, our methodology, and the input ingredients - the systematic effects of which are studied in this paper - are described. In Sect. 4, we discuss the consequences of varying the gas characteristics on the transport parameter determination. In Sect. 5, we focus on the effect of the source spectrum on the best-fit values for $\delta$ in models with or without convection/reacceleration. In Sect. 6, a similar analysis is carried out for the low-energy shape of the diffusion coefficient. We repeat again the analysis in Sect. 7, using various production cross-section sets. In a final step, we explore in Sect. 8 the effect of biasing B/C HEAO-3 data at high energy. We summarise, discuss our results, and conclude in Sect. 9.

\section{Description of the diffusion model}

The models and the equations are described in Paper II, to which we refer the reader for a complete discussion.

\subsection{Diffusion equation}

The differential density $N^{j}$ of the nucleus $j$ is a function of the total energy $E$ and the position $\boldsymbol{r}$ in the Galaxy. Assuming steadystate, the transport equation can be written in a compact form as

$\mathcal{L}^{j} N^{j}+\frac{\partial}{\partial E}\left(b^{j} N^{j}-c^{j} \frac{\partial N^{j}}{\partial E}\right)=\mathcal{S}^{j}$.

The operator $\mathcal{L}$ (we omit the superscript $j$ ) describes the diffusion $K(\boldsymbol{r}, E)$ and convection $\boldsymbol{V}(\boldsymbol{r})$ in the Galaxy, the decay rate $\Gamma_{\text {rad }}(E)=1 /\left(\gamma \tau_{0}\right)$ for radioactive species, and the destruction rate $\Gamma_{\text {inel }}(\boldsymbol{r}, E)=\sum_{\mathrm{ISM}} n_{\mathrm{ISM}}(\boldsymbol{r}) v \sigma_{\text {inel }}(E)$ on the interstellar matter (ISM):

$\mathcal{L}(\boldsymbol{r}, E)=-\boldsymbol{\nabla} \times(K \boldsymbol{\nabla})+\boldsymbol{\nabla} \cdot \boldsymbol{V}+\Gamma_{\text {rad }}+\Gamma_{\text {inel }}$.

The coefficients $b$ and $c$ are respectively first and second order gains/losses in energy, with

$$
\begin{aligned}
b(\boldsymbol{r}, E)= & \left\langle\frac{\mathrm{d} E}{\mathrm{~d} t}\right\rangle_{\text {ion, coul. }}-\frac{\boldsymbol{\nabla} \cdot \boldsymbol{V}}{3} E_{k}\left(\frac{2 m+E_{k}}{m+E_{k}}\right) \\
& +\frac{\left(1+\beta^{2}\right)}{E} \times K_{\mathrm{pp}}, \\
c(\boldsymbol{r}, E)= & \beta^{2} \times K_{\mathrm{pp}} .
\end{aligned}
$$

The coefficient $K_{\mathrm{pp}}$ is the diffusion coefficient in momentum space, and it can take several forms (see later).

\subsection{Geometry of the Galaxy}

The Galaxy is modelled to be a thin disc of half-thickness $h$, which contains the gas and the sources of CRs. This disc is embedded in a cylindrical diffusive halo of half-thickness $L$ (where the gas density is assumed to be equal to 0 ). A constant wind $\boldsymbol{V}(\boldsymbol{r})=\operatorname{sign}(z) \cdot V_{\mathrm{c}} \times \boldsymbol{e}_{z}$, perpendicular to the Galactic plane, is assumed. In this framework, CRs diffuse in the disc and in the halo independently of their position. These assumptions allow for semi-analytical solutions of the transport equation, as the interactions (destruction, spallations, energy gain and losses) are restricted to the thin disc. Such semi-analytical models reproduce all salient features of full numerical approaches (e.g., Strong \& Moskalenko 1998).
In this study, the disc half-height is set to $h=100 \mathrm{pc}$. It is not a physical parameter per se in the thin-disc approximation, but the phenomena occurring in the thin disc are related to it. The physical parameter is the surface density $\Sigma$ : should a different $h$ value be used, a rescaling always allows obtaining the same $\Sigma$.

Considering the radial extension $R$ of the Galaxy to be infinite leads to the $1 \mathrm{D}$ version of the DM. This geometry, used in Jones et al. (2001) and in our Paper II, is also used in this analysis. The corresponding sets of equations and their solutions are presented in the Appendix of Paper II. They are not repeated here.

\section{Methodology}

As in Papers I and II, three different classes of diffusion models are considered. In addition, for completeness, we also treat the pure diffusion case:

- Model $0=\left\{K_{0}, \delta\right\}$, i.e. pure diffusion $\left(V_{\mathrm{a}}=V_{\mathrm{c}}=0\right)$;

- Model I $=\left\{K_{0}, \delta V_{\mathrm{c}}\right\}$, i.e. no reacceleration $\left(V_{\mathrm{a}}=0\right)$;

- Model II $=\left\{K_{0}, \delta, V_{\mathrm{a}}\right\}$, i.e. no convection $\left(V_{\mathrm{c}}=0\right)$;

- Model III $=\left\{K_{0}, \delta, V_{\mathrm{c}}, V_{\mathrm{a}}\right\}$.

The parameters $K_{0}$ and $\delta$ come from the standard form assumed for the diffusion coefficient, namely,

$K(E)=\beta K_{0} \mathcal{R}^{\delta}$.

\subsection{Constrained parameters}

At most, for Model III, four transport parameters need to be determined from CR data:

- $K_{0}$, the normalisation of the diffusion coefficient (in unit of $\mathrm{kpc}^{2} \mathrm{Myr}^{-1}$ );

$-\delta$, the slope of the diffusion coefficient;

- $V_{\mathrm{c}}$, the constant convective wind perpendicular to the disc $\left(\mathrm{km} \mathrm{s}^{-1}\right)$;

- $V_{\mathrm{a}}$, the Alfvénic speed $\left(\mathrm{km} \mathrm{s}^{-1}\right)$ regulating the reacceleration strength [see Eq. (6)].

As in other studies, we use the $\mathrm{B} / \mathrm{C}$ ratio to constrain these parameters. In DMs, the halo size of the Galaxy $L$ is an extra free parameter. It cannot be determined solely from the $\mathrm{B} / \mathrm{C}$ ratio because of the well-known degeneracy between $K_{0}$ and $L$. In this study, we choose to fix $L$. The value of the transport parameters for any other values of $L$ can be obtained from simple scaling laws, as presented in Fig. 5 of Paper II (note that $\delta$ does not depend on $L$ ). To keep the discussion as simple as possible, this paper is based on $\mathrm{B} / \mathrm{C}$ data alone, with four free parameters $\left\{K_{0}, \delta, V_{\mathrm{c}}, V_{\mathrm{a}}\right\}$ and the halo size set to $L=4 \mathrm{kpc}$.

\subsection{Inputs and default configuration}

The inputs that we examine and vary are the following (details and references are given in Paper II, Sect. 2.4):

- gas surface density $\Sigma_{\mathrm{ISM}}=2 h n_{\mathrm{ISM}}$ and hydrogen fraction $f_{\mathrm{H}}$ (in number);

- source spectrum $Q^{j}(E)=q_{j} \beta^{\eta_{\mathrm{s}}} R^{-\alpha}\left(q_{j}\right.$ is scaled to match the measured elemental fluxes at $\sim 10 \mathrm{GeV} / \mathrm{n})$;

- spatial/momentum diffusion coefficients using different turbulence models (Schlickeiser 2002);

- production cross-sections. 
Table 1. Reference values for the inputs.

\begin{tabular}{ll}
\hline \hline Input name & Default value/dependence/set \\
\hline Gas & $\Sigma=6.17 \times 10^{20} \mathrm{~cm}^{2}, \quad f_{\mathrm{H}}=90 \%$ \\
Source spectrum & $\eta_{\mathrm{S}}=-1, \quad \alpha+\delta=2.65$ \\
$K(E)$ and $K_{\mathrm{pp}}(E)$ & Slab Alfvén (SA): Eqs. (5) \& (6) \\
Cross-sections & W03 (Webber et al. 2003) \\
Data & $\mathrm{B} / \mathrm{C}$, dataset $\mathrm{F}^{\dagger}$ \\
\hline
\end{tabular}

Notes. In subsequent tables of the paper, all results obtained with the default inputs are in italics. ${ }^{(\dagger)} 31$ data points from IMP7-8, Voyager $1 \& 2$, ACE, HEA0-3, Spacelab, and CREAM04.

The reference (default) values for these inputs are gathered in Table 1. The default diffusion coefficient $K_{\mathrm{pp}}$ (in momentum space) is taken from the model of minimal reacceleration by the interstellar turbulence (Osborne \& Ptuskin 1988; Seo \& Ptuskin 1994):

$K_{\mathrm{pp}} \times K=\frac{4}{3} V_{\mathrm{a}}^{2} \frac{p^{2}}{\delta\left(4-\delta^{2}\right)(4-\delta)}$,

where $V_{\mathrm{a}}$ is the Alfvénic speed in the medium.

Concerning the experimental data used to fit the $\mathrm{B} / \mathrm{C}$ ratio, as in Paper II, we use the following dataset (denoted $\mathrm{F}$ in Paper II), which consists of $31 \mathrm{~B} / \mathrm{C}$ data points (see Fig. 5 of this paper): IMP7-8 (Garcia-Munoz et al. 1987), Voyager $1 \& 2$ (Lukasiak et al. 1999), ACE-CRIS (de Nolfo et al. 2006), HEA03 (Engelmann et al. 1990), Spacelab (Swordy et al. 1990), and CREAM (Ahn et al. 2008).

\subsection{Minimisation routine}

In Papers I and II, we adapted the MCMC technique for a propagation code. This allows the PDF of the parameters to be obtained along with their statistical uncertainties. However, this technique relies on thousands of calculations for a single setting of the inputs, which is not optimal for speed. Here, we are only interested in the best-fit values, not in the PDF of the parameters. Therefore, we use the Minuit library (a CERN library), which provides minimisation routines. Instead of a few hours of distributed calculations for the MCMC technique, a few minutes on a workstation are enough to obtain the best-fit parameters.

A few configurations have been cross-checked with the MCMC technique, to ensure that the typical widths of the PDFs remain the same, whatever the input ingredients used in the calculation. This allows us, for a given propagation model calculated from different input ingredients, to compare the resulting scatter in the transport parameter values - systematic uncertainties (hereafter SystUnc) - to the typical statistical uncertainties (hereafter StatUnc) found with the MCMC technique.

\section{Influence of the gas description}

We start by varying the gas parameters. This is discussed for the most general class of the DM, i.e. Model III (allowing for both convection and reacceleration). For obvious reasons (see below), our conclusions also hold for Models 0, I, or II.

In 1D models, the surface density of the gas in the model corresponds to the average of the true gas surface density (which depends on the position in the Galaxy) over the effective diffusion volume (Taillet \& Maurin 2003). This is a reasonable assumption to make for stable nuclei (see Paper II). However, the
Table 2. Best-fit transport parameters for different ISM.

\begin{tabular}{ccccccc}
\hline \hline \multicolumn{2}{c}{ Gas } & $\begin{array}{c}K_{0}^{\text {best }} \times 10^{2} \\
\left(\mathrm{kpc}^{2} \mathrm{Myr}^{-1}\right)\end{array}$ & $\delta^{\text {best }}$ & $\begin{array}{c}V_{\mathrm{c}}^{\text {best }} \\
\left(\mathrm{km} \mathrm{s}^{-1}\right)\end{array}$ & $\begin{array}{c}V_{\mathrm{a}}^{\text {best }} \\
\left(\mathrm{km} \mathrm{s}^{-1}\right)\end{array}$ & $\chi^{2} /$ d.o.f. \\
\hline$\Sigma^{\text {ref }}$ & $f_{\mathrm{H}}^{\text {ref }}$ & 0.48 & 0.86 & 18.8 & 38.0 & 1.47 \\
$\Sigma^{\text {ref }}$ & $95 \%$ & 0.53 & 0.83 & 18.6 & 38.1 & 1.24 \\
$\Sigma^{\text {ref }}$ & $80 \%$ & 0.41 & 0.90 & 19.3 & 37.7 & 2.14 \\
$\frac{1}{2} \Sigma^{\text {ref }}$ & $f_{\mathrm{H}}^{\text {ref }}$ & 0.25 & 0.85 & 9.5 & 19.4 & 1.45 \\
$2 \Sigma^{\text {ref }}$ & $f_{\mathrm{H}}^{\text {ref }}$ & 0.92 & 0.86 & 37.3 & 74.6 & 1.51 \\
\hline
\end{tabular}

Notes. Model III for $L=4 \mathrm{kpc}$. For all settings, we keep fixed $\left\langle n_{\mathrm{e}^{-}}\right\rangle=$ 0.033 and $T_{\mathrm{e}} \sim 10^{4} \mathrm{~K}$. The reference parameters for the surface density $\Sigma^{\text {ref }}$ and the hydrogen fraction $f_{\mathrm{H}}^{\text {ref }}$ are given in Table 1.

details of the volume over which to calculate this average are not straightforward. Moreover, even if a more realistic distribution of gas were to be used, the latter is not free of uncertainties. We thus allow for some uncertainty in this input. We also vary the fraction of hydrogen relative to helium.

\subsection{Influence of the hydrogen fraction}

In Table 2, the second and third lines (compare with the first line) show the effect of changing the hydrogen fraction in the ISM: the transport parameters are changed at most by $\sim 5 \%$. There is a systematic trend for $\delta$ to decrease with smaller fractions of helium in the gas. However, the uncertainty on the hydrogen and helium fraction is not more than a few $\%$. We therefore conclude that this has no strong impact on the derived transport parameters.

\subsection{Influence of the surface density}

The fourth and fifth lines show the effect of changing the surface gas density $\Sigma_{\text {ISM }}=2 h n_{\text {ISM }}$. Whereas it has a small impact on the diffusion slope $\delta$, the other transport parameters are strongly affected. The change in the parameters can be understood if we look at the grammage of the DM (Maurin et al. 2002). In the purely diffusive regime, we have (e.g., Maurin et al. 2006)

$\langle x\rangle^{\text {pure-DM }}=\frac{\Sigma_{\mathrm{ISM}} \bar{m} v L}{2 K}$,

where $\bar{m}$ is the mean mass of the ISM. Let $K_{0}^{\mathrm{ref}}, \delta^{\mathrm{ref}}, V_{\mathrm{c}}^{\mathrm{ref}}$, and $V_{\mathrm{a}}^{\text {ref }}$ be the best-fit parameters obtained for a surface density of gas $\Sigma^{\text {ref }}$ (first line of the table). We remind that $L$ is fixed here. If the surface gas density is rescaled, i.e. $\Sigma^{\text {new }}=x \times \Sigma^{\text {ref }}$, in order to keep the same grammage in Eq. (7), we need to have $K_{0}^{\text {new }}=x \times K_{0}^{\text {ref }}$. This is what we get in Table 2 .

The same reasoning holds for the convective wind. In presence of a constant wind, the full expression for the grammage reads (e.g., Maurin et al. 2006)

$\langle x\rangle^{V_{\mathrm{c}}} \equiv \frac{\Sigma_{\mathrm{ISM}} \bar{m} v}{2 V_{\mathrm{c}}}\left[1-\mathrm{e}^{-\frac{V_{\mathrm{c} L}}{K}}\right]$.

With the above rescaling for $\Sigma^{\text {new }}$ and $K_{0}^{\text {new }}$, we get $V_{c}^{\text {new }}=x \times$ $V_{\mathrm{c}}^{\mathrm{ref}}$. Again, this is recovered in Table 2.

The last parameter is the Alfvénic speed, for which we need to consider the whole transport equation. The speed $V_{\mathrm{a}}$ is used to define $K_{\mathrm{pp}}$ (see Eq. (6)), which appears in the terms $b$ and $c$ (Eqs. (3) and (4)) of Eq. (1). If we consider the latter equation and the transport operator $\mathcal{L}$ in Eq. (2), the rescaling $\Sigma^{\text {new }}=$ $x \times \Sigma^{\text {ref }}$ leads to $\mathcal{L}^{\text {new }}=x \times \mathcal{L}^{\text {ref }}$. This implies $b^{\text {new }}=x \times b^{\text {ref }}$, 
Table 3. Best-fit transport parameters for different slopes $\gamma$.

\begin{tabular}{lccccc}
\hline \hline$\gamma$ & $\begin{array}{c}K_{0}^{\text {best }} \times 10^{2} \\
\left(\mathrm{kpc}^{2} \mathrm{Myr}^{-1}\right)\end{array}$ & $\delta^{\text {best }}$ & $\begin{array}{c}V_{\mathrm{c}}^{\text {best }} \\
\left(\mathrm{km} \mathrm{s}^{-1}\right)\end{array}$ & $\begin{array}{c}V_{\mathrm{a}}^{\text {best }} \\
\left(\mathrm{km} \mathrm{s}^{-1}\right)\end{array}$ & $\chi^{2} /$ d.o.f. \\
\hline 0: 2.55 & 4.13 & 0.39 & $\ldots$ & $\ldots$ & 29.7 \\
O: 2.65 & 4.08 & 0.40 & $\ldots$ & $\ldots$ & 28.8 \\
0: 2.75 & 4.02 & 0.40 & $\ldots$ & $\ldots$ & 27.9 \\
0: 2.85 & 3.97 & 0.40 & $\ldots$ & $\ldots$ & 27.0 \\
I: 2.55 & 0.42 & 0.93 & 14.0 & $\ldots$ & 11.4 \\
I: 2.65 & 0.42 & 0.93 & 13.5 & $\ldots$ & 11.2 \\
I: 2.75 & 0.42 & 0.93 & 13.1 & $\ldots$ & 11.0 \\
I: 2.85 & 0.42 & 0.93 & 12.7 & $\ldots$ & 10.9 \\
II: 2.55 & 9.41 & 0.24 & $\ldots$ & 74.2 & 4.52 \\
II: 2.65 & 9.76 & 0.23 & $\ldots$ & 73.1 & 4.73 \\
II: 2.75 & 10.1 & 0.23 & $\ldots$ & 72.1 & 4.94 \\
II: 2.85 & 10.4 & 0.22 & $\ldots$ & 71.0 & 5.15 \\
& & & & & \\
III: 2.55 & 0.52 & 0.84 & 18.9 & 39.7 & 1.25 \\
III: 2.65 & 0.48 & 0.86 & 18.8 & 38.0 & 1.47 \\
III: 2.75 & 0.45 & 0.87 & 18.8 & 36.4 & 1.75 \\
III: 2.85 & 0.42 & 0.88 & 18.6 & 35.0 & 2.10 \\
\hline
\end{tabular}

Notes. Models 0, I, II, and III for $L=4 \mathrm{kpc}$. The parameter $\gamma$ is the slope of the propagated fluxes $(\gamma=\alpha+\delta)$. The source spectrum is $Q(E) \propto \beta^{\eta_{\mathrm{S}}} \mathcal{R}^{-\alpha}$, and $\eta_{\mathrm{S}}=-1$.

$c^{\text {new }}=x \times c^{\text {ref }}$, and $\mathcal{S}^{\text {new }}=x \times \mathcal{S}^{\text {ref }}$. Let us consider the three terms each in turn. For $b$, if we look at Eq. (3), this $x$ factor is automatically ensured for the Coulomb and ionisation losses (they depend on the gas surface density), and also for adiabatic losses (as $V_{\mathrm{c}}^{\text {new }}=x \times V_{\mathrm{c}}^{\mathrm{ref}}$ ). This implies $K_{\mathrm{pp}}^{\text {new }}=x \times K_{\mathrm{pp}}^{\mathrm{ref}}$. From Eq. (6), we have $K_{\mathrm{pp}} \propto V_{\mathrm{a}}^{2} / K_{0}$, such that the previous equality yields

$\left(V_{\mathrm{a}}^{\text {new }}\right)^{2} / K_{0}^{\text {new }}=x \times\left(V_{\mathrm{a}}^{\text {ref }}\right)^{2} / K_{0}^{\text {ref }}$.

This gives $V_{\mathrm{a}}^{\text {new }}=x \times V_{\mathrm{a}}^{\text {ref }}$ (the same information is given by the $c$ term), in agreement with the results of Table 2 .

\subsection{Summary for the gas surface density}

An uncertainty of $x \%$ on the gas surface density leads to SystUnc of $x \%$ on $K_{0}, V_{\mathrm{c}}$ and $V_{\mathrm{a}}$, but it leaves the diffusion slope $\delta$ unchanged. This is true, whatever the value for the halo size $L$. For the sake of completeness, we should note that this also affects the source term: the source abundances are now $q^{\text {new }}=x \times q^{\text {ref }}$.

\section{Influence of the source spectrum}

In Paper I, we showed that some correlations exist between the source spectra and the transport parameters. Below, we investigate in more detail how the transport parameters are changed if we assume different input for $\alpha$ (spectral index of the sources) and $\eta_{\mathrm{S}}$ (related to the spectral shape at low energy:

$Q(E) \propto \beta^{\eta_{\mathrm{S}}} \mathcal{R}^{-\alpha}$.

\subsection{Influence of the source slope $\alpha$}

It is usually said that the source spectrum energy dependence $Q(E)$ factors out of the secondary-to-primary ratio. This agrees with the results gathered in Table 3 , which shows the effect of
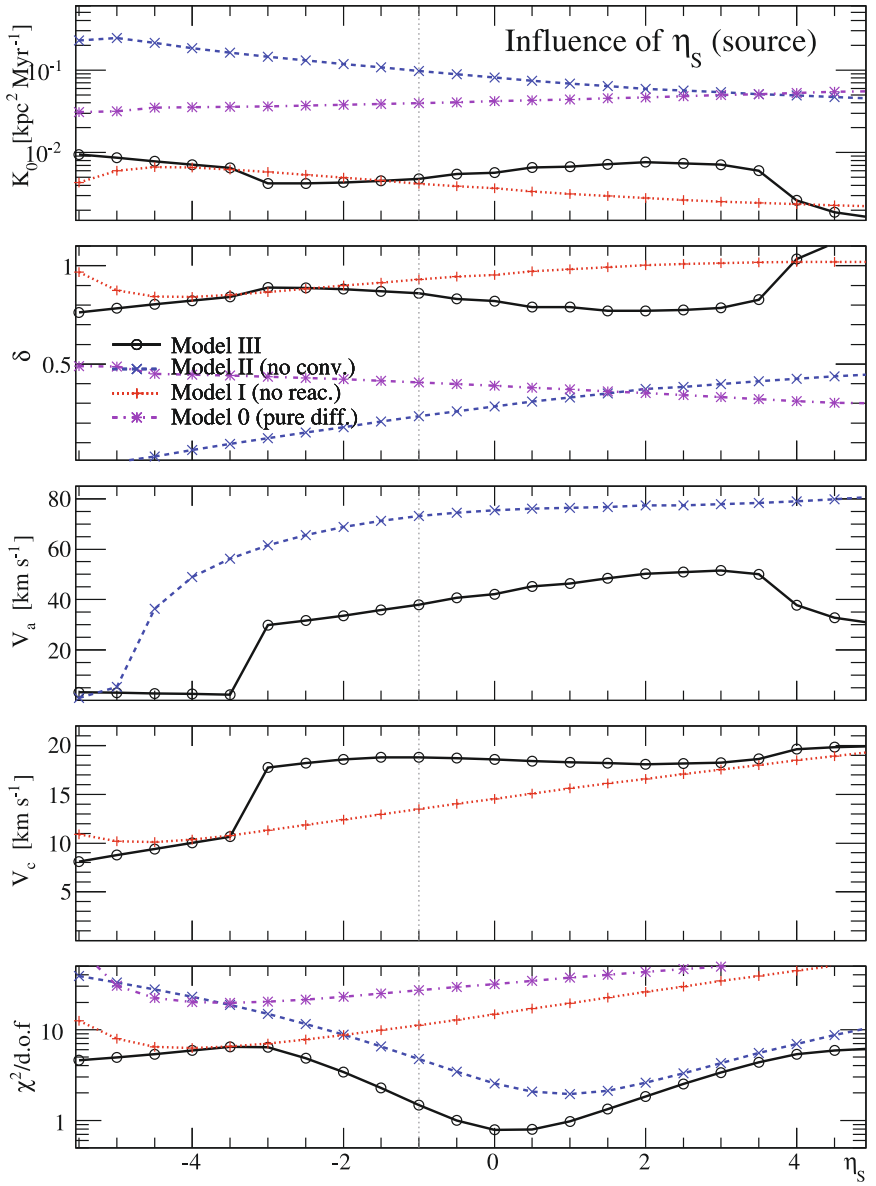

Fig. 1. From top to bottom: best-fit values of $K_{0}, \delta, V_{\mathrm{a}}, V_{\mathrm{c}}$, and the associated $\chi^{2} /$ d.o.f. as a function of $\eta_{\mathrm{S}}$ [see Eq. (9)]. Here, $\gamma=\alpha+\delta=2.65$. The vertical grey-dotted line is a guideline for the results at the default configuration $\eta_{\mathrm{S}}=-1$.

varying the source slope $\alpha$ (or equivalently the slope of the measured fluxes $\gamma=\alpha+\delta$ ). This is especially true for Model I (no reacceleration), whereas if reacceleration is included (Models II and III), a few $\%$ change on the parameter $\gamma$ leads to a few $\%$ change on $V_{\mathrm{a}}, K_{0}$, and $\delta$.

From the last column of the same table, we see that on a statistical basis, the best model is Model III, then Model II, Model I, and finally Model 0 . The relative merit (goodness-of-fit) of each class of models (III, II, I, and 0) remains unchanged when $\alpha$ is changed.

\subsection{Influence of the low-energy source spectrum through $\eta_{\mathrm{S}}$}

The second parameter that can be varied in the source term is the low-energy shape $\beta^{\eta_{\mathrm{s}}}$ [see Eq. (9)], which is a priori not very well constrained, both from observational and theoretical points of view. The evolution of the best-fit transport parameters as a function of $\eta_{\mathrm{S}}$ is shown in Fig. 1, for Model 0 (dash-dotted lines/stars), I (dotted lines/plusses), II (dashed lines/crosses), and III (solid lines/open circles).

The three configurations have marked minima in their $\chi_{\min }^{2}$ (bottom panel), but not at the same $\eta_{\mathrm{S}}$. Models 0 and I have a similar $\chi^{2}$ dependence (weak impact of the presence of the wind), as have Models II and III (both affected by reacceleration). Understanding the exact dependence of the transport parameters with $\eta_{\mathrm{S}}$ is not the goal of the analysis. The important 
D. Maurin et al.: Systematic uncertainties on the cosmic-ray transport parameters

Table 4. Best-fit values from a simultaneous fit on $\mathrm{B} / \mathrm{C}$ and $\mathrm{O}$.

\begin{tabular}{lcccc}
\hline \hline Model & $\eta_{\mathrm{S}}^{(\mathrm{B} / \mathrm{C}+\mathrm{O})}$ & $\alpha^{(\mathrm{B} / \mathrm{C}+\mathrm{O})}$ & $\delta^{(\mathrm{B} / \mathrm{C}+\mathrm{O})}$ & $\chi_{\min }^{2} /$ d.o.f. \\
\hline 0 & -0.82 & 2.22 & 0.41 & 25.3 \\
I & -1.23 & 2.23 & 0.98 & 9.23 \\
II & 1.24 & 2.24 & 0.35 & 3.62 \\
III & 1.56 & 2.30 & 0.95 & 2.31 \\
\hline
\end{tabular}

Notes. The data used are IMP7-8, Voyager 1\&2, ACE, HEA0-3, Spacelab, and CREAM04 for the B/C ratio, and HEAO-3 for the oxygen flux.

point to underline is that changing $\eta_{\mathrm{S}}$ does not allow reconciling convection Models I and III with smaller $\delta$. As $\delta$ varies only in the range $0.3-0.5$ for Model 0 (pure diffusion), we conclude that for present $\mathrm{B} / \mathrm{C}$ data, large $\delta$ are associated to the constant Galactic wind. The largest variation is observed for Model II (reacceleration only), for which $\delta$ is allowed to be either smaller or larger than $\delta_{\mathrm{II}}^{\text {best }}=0.33$ (associated with $\eta_{\mathrm{S}}^{\text {best }} \approx 1$ ) for a smaller or a larger $\eta_{\mathrm{S}}$. For any $\eta_{\mathrm{S}}$, Model III always performs better than the other two models.

\subsection{Fitting $\alpha$ and $\eta_{\mathrm{S}}$ using a primary flux}

The source parameters $\alpha$ and $\eta_{\mathrm{S}}$ are not completely free. They can be fitted directly on primary elemental fluxes (see Paper I). If we determine simultaneously the transport and source parameters from a fit on $\mathrm{B} / \mathrm{C}+\mathrm{O}$ HEAO-3 data, we obtain the best-fit values gathered in Table 4. For Models II and III, $\eta_{\mathrm{S}}^{\mathrm{B} / \mathrm{C}+\mathrm{O}}$ is not far from $\eta_{\mathrm{S}}^{\text {best }}$ found from the B/C fit (see Fig. 1). For Model II, using the above constraint $\left(\eta_{\mathrm{S}}^{\mathrm{B} / \mathrm{C}+\mathrm{O}}=1.24\right)$ selects the diffusion slope $\delta^{\mathrm{B} / \mathrm{C}+\mathrm{O}}=0.35$, close to the Kolmogorov spectrum for the turbulence.

The surprising result is that the value for $\alpha^{\mathrm{B} / \mathrm{C}+\mathrm{O}}$ is quite resilient to any slope obtained for $\delta$. This is odd, since it leaves the propagated slope $\gamma=\alpha+\delta$ quite unconstrained, leading to either 2.6 (Model II) or 3.2 (Model III). This indicates that in Model III, pure diffusive transport (i.e. $\gamma=\alpha+\delta$ ) has not yet been reached at $\mathrm{TeV} / \mathrm{n}$ energies.

\subsection{Summary for the source effect}

The dependence of the transport parameters with the source parameters $\alpha$ and $\eta_{\mathrm{S}}$ are very different. We checked that the transport parameters do not significantly depend on the source parameter $\alpha$ (in particular, $\delta$ is unaffected). Still, correlations exist between the source and the other transport parameters (see Paper I), and from Table 3, we can estimate that they affect their best-fit values at most by a few ten percent.

On the other hand, Fig. 1 shows that the transport parameters (including $\delta$ ) are quite sensitive to the low-energy source spectrum parameterised by $\eta_{\mathrm{S}}$. In any case, this does not allow reconciliation of Model III with small $\delta$. Taking a sizeable range for $\eta_{\mathrm{S}}$ always lead to $\delta \gtrsim 0.6$. This is even worse for Model I (no reacceleration). At variance, Model II (no convection) is very sensitive to $\eta_{\mathrm{S}}$. However, $\eta_{\mathrm{S}}$ is constrained by primary fluxes measurements, and by no means could it span the range shown in Fig. 1. When this constraint is considered, the best-fit $\delta$ for Model II turns out to be 0.35 . Given the sensitivity of the latter to $\eta_{\mathrm{S}}$ and the possible bias in low-energy data (because of the solar modulation), this is consistent with a Kolmogorov spectrum. If the constraint of the primary flux is taken into account, the
Table 5. $K(E)$ and $K_{\mathrm{pp}}$ for different schemes.

\begin{tabular}{llcl}
\hline \hline \multicolumn{2}{l}{ Type of turbulence } & $\eta_{\mathrm{T}}$ & $\frac{K_{\mathrm{pp}} K_{\mathrm{xx}}}{4 / 3 p^{2} V_{\mathrm{a}}^{2}}$ \\
\hline LBI & Leaky Box Inspired & 0 & $\frac{1}{\delta\left(4-\delta^{2}\right)(4-\delta)}$ \\
SA & Slab Alfvén & 1 & $\frac{1}{\delta\left(4-\delta^{2}\right)(4-\delta)}$ \\
IFM & Isotropic fast magnetosonic & $2-\delta$ & $\beta^{1-\delta} \ln \left(\frac{v}{V_{\mathrm{a}}}\right)$ \\
Mix & Mixture SA and IFM & $1-\delta$ & $\beta^{1-\delta} \ln \left(\frac{v}{V_{\mathrm{a}}}\right)$ \\
\hline
\end{tabular}

Notes. The spatial diffusion coefficient is $K_{\mathrm{xx}}=\beta^{\eta_{\mathrm{T}}} \cdot K_{0} \cdot \mathcal{R}^{\delta}$.

uncertainty on the transport parameters (associated to the source parameter $\eta_{\mathrm{S}}$ ) can be estimated as $\sim 10-20 \%$.

The puzzling point is that, although Model III is preferred over Model II on a statistical basis, the latter seems to agree more with the expected theoretical value of $\delta$. This is more of further if we consider the quantity $\gamma=\alpha+\delta$, the asymptotic slope reached in the purely diffusive regime. The TRACER experiment finds $\gamma \approx 2.65$ for all nuclei (Ave et al. 2008), whereas the best-fit slope from high-energy $\mathrm{H}$ and $\mathrm{He}$ data is $\gamma \approx 2.85$ (Donato et al. 2009). From the propagated slope's point of view, Model II is again favoured over Model III, since the former leads to $\gamma=2.59$, whereas the latter leads to 3.25 (read off Table 4). If Model III is confirmed, as already emphasised above, this would mean that even high-energy data have not reached the purely diffusive transport yet.

\section{Influence of the low-energy diffusion coefficient}

We now turn to the effect of the low-energy shape of the diffusion coefficient. Assuming different diffusion schemes lead to different forms of the spatial and momentum diffusion coefficient (Schlickeiser 2002). Recently, Ptuskin et al. (2006) also argue that the form of the spatial diffusion coefficient can change at low energy, due to the possibility that the nonlinear MHD cascade sets the power-law spectrum of turbulence.

We first consider several theoretically-motivated diffusion forms given in the literature. They mostly differ at low energy, so that it is useful to rewrite Eq. (5) as

$K(E)=\beta^{\eta_{\mathrm{T}}} \cdot K_{0} \mathcal{R}^{\delta}$.

In the second step, we let $\eta_{\mathrm{T}}$ vary over a wide range in order to draw more general conclusions.

\subsection{Influence of the turbulence scheme}

A few turbulence schemes are gathered in Table 5. The associated best-fit values of the transport coefficients are presented in Table 6. When only convection is present (Model I), the lowenergy form of $K(E)$ is irrelevant, as seen in Table 6. For models with reacceleration (Models II and III), it significantly affects almost all parameters. If there is no wind (Model II), the effect is maximum on $\delta$. The model with pure diffusion (Model 0 ), or with both convection and reacceleration (Model III), falls inbetween. For Model III, the cases SA and IFM, on the one hand, and LBI and Mix, on the other, give very similar results. This is easily understood as the quantities $\eta_{\mathrm{T}}^{\mathrm{SA}}=2-\delta$ and $\eta_{\mathrm{T}}^{\mathrm{IMF}}=1$ (respectively $\eta_{\mathrm{T}}^{\mathrm{LBI}}=1-\delta$ and $\eta_{\mathrm{T}}^{\mathrm{Mix}}=0$ ) are roughly equal for $\delta_{\mathrm{III}}^{\text {best }} \sim 1$.

The important result is that Model III is mildly sensitive to the diffusion scheme, with an uncertainty of a few ten percent 
Table 6. Best-fit transport parameters based on different low-energy dependence of the diffusion coefficient.

\begin{tabular}{lccccc}
\hline \hline Type & $\begin{array}{c}K_{0}^{\text {best }} \times 10^{2} \\
\left(\mathrm{kpc}^{2} \mathrm{Myr}^{-1}\right)\end{array}$ & $\delta^{\text {best }}$ & $\begin{array}{c}V_{\mathrm{c}}^{\text {best }} \\
\left(\mathrm{km} \mathrm{s}^{-1}\right)\end{array}$ & $\begin{array}{c}V_{\mathrm{a}}^{\text {best }} \\
\left(\mathrm{km} \mathrm{s}^{-1}\right)\end{array}$ & $\chi^{2} /$ d.o.f \\
\hline 0: LBI & 3.48 & 0.45 & $\ldots$ & $\ldots$ & 17.5 \\
0: SA & 4.08 & 0.40 & $\ldots$ & $\ldots$ & 28.8 \\
0: IFM & 4.30 & 0.38 & $\ldots$ & $\ldots$ & 36.7 \\
0: Mix & 3.71 & 0.43 & $\ldots$ & $\ldots$ & 23.7 \\
I: LBI & 0.40 & 0.94 & 13.6 & $\ldots$ & 12.0 \\
I: SA & 0.42 & 0.93 & 13.5 & $\ldots$ & 11.2 \\
I: IFM & 0.42 & 0.93 & 13.5 & $\ldots$ & 11.6 \\
I: Mix & 0.41 & 0.94 & 13.5 & $\ldots$ & 12.0 \\
II: LBI & 5.50 & 0.38 & $\ldots$ & 65.0 & 1.61 \\
II: SA & 9.76 & 0.23 & $\ldots$ & 73.1 & 4.73 \\
II: IFM & 14.0 & 0.16 & $\ldots$ & 18.9 & 6.86 \\
II: Mix & 7.13 & 0.32 & $\ldots$ & 12.8 & 2.03 \\
III: LBI & 0.70 & 0.78 & 18.0 & 47.1 & 0.87 \\
III: SA & 0.48 & 0.86 & 18.8 & 38.0 & 1.47 \\
III: IFM & 0.49 & 0.85 & 18.9 & 45.6 & 1.25 \\
III: Mix & 0.73 & 0.77 & 17.8 & 57.4 & 0.93 \\
\hline
\end{tabular}

Notes. Models 0 , I, II, and III for $L=4 \mathrm{kpc}$. SA corresponds to the reference DM used throughout the paper.

scatter on all the transport parameters. However, Model II parameters are extremely sensitive to the diffusion scheme (more than a factor of 2 scatter). Depending on the case considered, $\delta$ is found in the range $0.16-0.38$. The hierarchy of $\chi_{\min }^{2}$ among the various models is always conserved.

\subsection{Generalisation to any $\eta_{\top}$}

We generalise the analysis by allowing for any value of the parameter $\eta_{\mathrm{T}}$ in the diffusion coefficient $K(E)$. In doing so, we do not seek to provide sound physical motivations for the range tested. In this section, whatever the value of $\eta_{\mathrm{T}}$, the diffusion coefficient in momentum space is assumed to follow $K_{\mathrm{pp}} K_{\mathrm{xx}}=$ $(4 / 3) p^{2} V_{\mathrm{a}}^{2} /\left(\delta\left(4-\delta^{2}\right)(4-\delta)\right)$.

The best-fit transport parameters and $\chi_{\min }^{2}$ evolution as a function of $\eta_{\mathrm{T}}$ are plotted in Fig. 2. For $\eta_{\mathrm{T}} \lesssim-2$, all the models converge slowly towards purely diffusive models (no convection, no reacceleration). But this is at the cost of a bad $\chi^{2}$ (see bottom panel). Based on the $\chi^{2}$ criterium, high values of $\eta_{\mathrm{T}}(\gtrsim 2)$ are also disfavoured. The four configurations have marked minima in their $\chi_{\min }^{2}$, corresponding to $\eta_{\mathrm{T}}^{\text {best }} \approx-2.75,-2.5,-0.25,+0.25$ for Models 0, I, II, and III respectively, for which $\delta^{\text {best }} \approx$ $0.6,0.6,0.4,0.8$. For $\eta_{\mathrm{T}} \sim-2.5$, all models point to $\delta \sim 0.5$, close to a Kraichnan spectrum for turbulence.

For Model III, for a well-chosen value of $\eta_{\mathrm{T}}$, the diffusion slope could be decreased at most down to $\delta=0.5$, but such a configuration does not correspond to the minimal $\chi^{2}$ for this model so is excluded. For Model II, almost any value of $\delta$ can be reached.

\subsection{Summary for the diffusion coefficient effect}

Similars to the effect of the source parameter $\eta_{\mathrm{S}}$, varying the parameter $\eta_{\mathrm{T}}$ on a wide range i) does not to allow $\delta \lesssim 1 / 2$ to reached for Model III, and ii) strongly affects $\delta$ for Model II. For
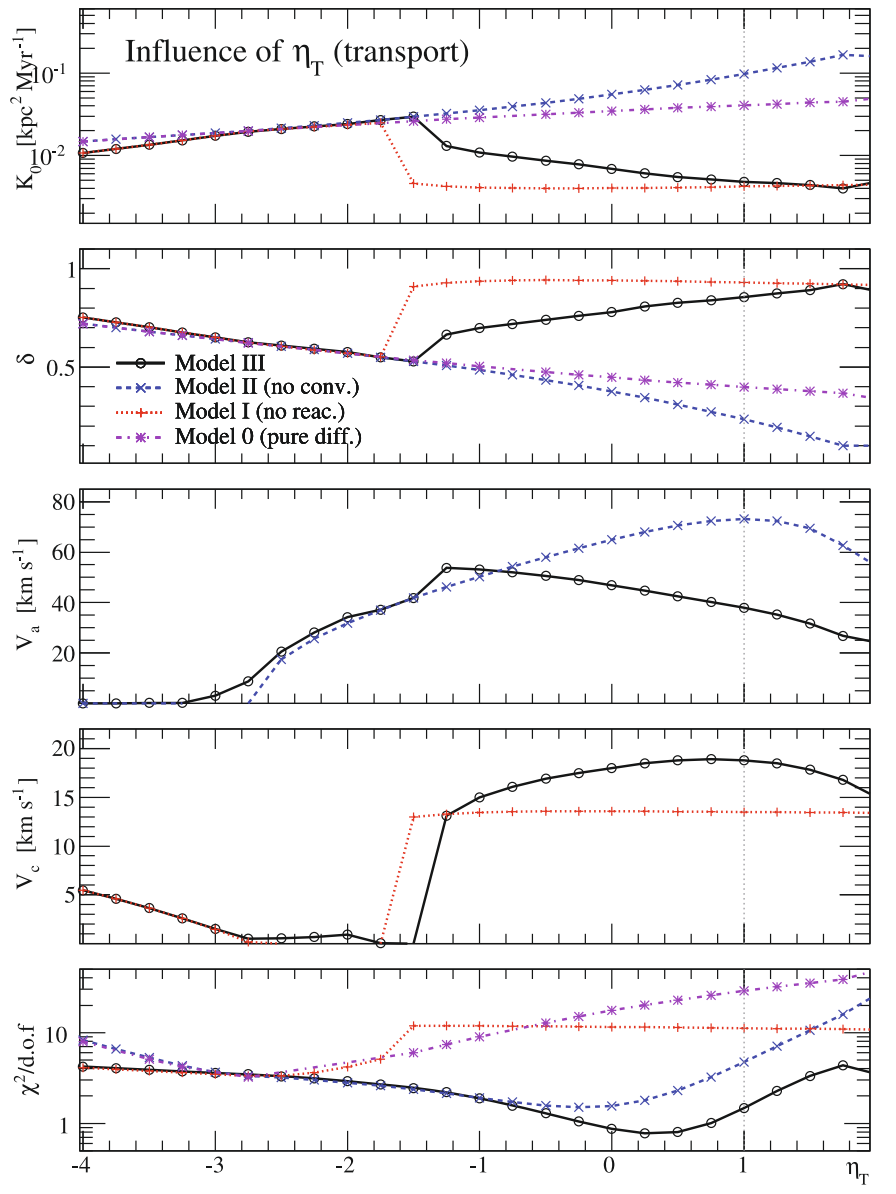

Fig. 2. Same as Fig. 1, but now as a function of $\eta_{\mathrm{T}}$ [see Eq. (10)]. The vertical grey-dotted line is for the default configuration $\eta_{\mathrm{T}}=1(\mathrm{SA})$.

the latter, the best-fit $\eta_{\mathrm{T}}$ leads to $\delta \approx 0.4$, slightly more than the Kolmogorov spectrum of turbulence.

From a statistical point of view, Model III is again preferred, except in the region $-3 \lesssim \eta_{\mathrm{T}} \lesssim-1.5$ where $V_{\mathrm{c}}$ drops to zero, so that it is equivalent to Model II. For $\eta_{\mathrm{T}} \lesssim-2.75, V_{\mathrm{a}}$ also drops to zero, so that all models are close to the purely diffusive case (Model 0), which favours $\delta \sim 1 / 2$. As before, one of the interesting features of Model II (reacceleration only) is its versatility. As the range of allowed $\eta_{\mathrm{T}}$ remains unspecified to some extent, Model II does not point to any specific value of $\delta$. It can accommodate values as low as $1 / 3$, going through $1 / 2$ and even higher values, if a sharp turn-off (negative values of $\eta_{\mathrm{T}}$ ) exists in the diffusion coefficient (e.g., Ptuskin et al. 2006).

\section{Influence of the production cross-sections}

Many reaction channels are required to calculate the production of secondary species. Semi-analytical formulae, semi-empirical approaches, or even fit to the data are currently used in the literature to obtain the full set of production cross-section in CR physics. We show first the different best-fit values obtained for different sets available in the literature. We then inspect what would be the effect of energy-biased cross-sections on the transport parameter derivation. 
Table 7. Best-fit transport parameters for various cross-section sets.

\begin{tabular}{lccccc}
\hline \hline X-files & $\begin{array}{c}K_{0}^{\text {best }} \times 10^{2} \\
\left(\mathrm{kpc}^{2} \mathrm{Myr}^{-1}\right)\end{array}$ & $\delta^{\text {best }}$ & $\begin{array}{c}V_{\mathrm{c}}^{\text {best }} \\
\left(\mathrm{km} \mathrm{s}^{-1}\right)\end{array}$ & $\begin{array}{c}V_{\mathrm{a}}^{\text {best }} \\
\left(\mathrm{km} \mathrm{s}^{-1}\right)\end{array}$ & $\chi^{2} /$ d.o.f. \\
\hline 0: WKS98 & 2.66 & 0.53 & $\ldots$ & $\ldots$ & 23.5 \\
0: S01 & 3.40 & 0.40 & $\ldots$ & $\ldots$ & 34.5 \\
0: W03 & 4.08 & 0.40 & $\ldots$ & $\ldots$ & 28.8 \\
0: GAL09 & 3.83 & 0.46 & $\ldots$ & $\ldots$ & 28.4 \\
I: WKS98 & 0.45 & 0.95 & 10.4 & $\ldots$ & 12.0 \\
I: S01 & 0.25 & 1.01 & 11.9 & $\ldots$ & 13.5 \\
I: W03 & 0.42 & 0.93 & 13.5 & $\ldots$ & 11.2 \\
I: GAL09 & 0.49 & 0.95 & 13.6 & $\ldots$ & 12.3 \\
II: WKS98 & 7.19 & 0.31 & $\ldots$ & 71.5 & 3.40 \\
II: S01 & 9.27 & 0.22 & $\ldots$ & 68.8 & 6.56 \\
II: W03 & 9.76 & 0.23 & $\ldots$ & 73.1 & 4.73 \\
II: GAL09 & 10.0 & 0.26 & $\ldots$ & 85.0 & 4.03 \\
III: WKS98 & 0.69 & 0.80 & 15.8 & 43.5 & 1.11 \\
III: S01 & 0.25 & 0.98 & 16.2 & 27.9 & 3.01 \\
III: W03 & 0.48 & 0.86 & 18.8 & 38.0 & 1.47 \\
III: GAL09 & 0.65 & 0.82 & 21.7 & 49.4 & 1.53 \\
\hline
\end{tabular}

Notes. Models 0, I, II, and III for $L=4 \mathrm{kpc}$. The four base sets of cross-sections are WKS98 from Webber et al. (1998), S01 from a private communication of Aimé Soutoul (2001), W03 from Webber et al. (2003), and GAL09 from the GALPROP code v50.1p (2009).

\subsection{Using different sets of fragmentation cross-sections}

We use four different sets of cross-sections. Among them, the most up-to-date are W03 (Webber et al. 2003) and GAL09 (from the GALPROP $\operatorname{code}^{1}$ ). The former is based on the semiempirical approach of Webber and coworkers, initiated in the 90's (Webber et al. 1990). The latter set takes advantage of the former approach, renormalising some cross-sections to selected data. The two others, WKS98 (Webber et al. 1998) and S01 (private communication of Aimé Soutoul 2001), are a bit outdated, but they serve as an illustration here.

The effect of these different sets on the parameter determination is shown in Table 7. The scatter in the values of the different parameters is not the same depending on the model considered. WKS98 requires less convection than any other set, and GAL09 needs more reacceleration than the others. Otherwise, it is difficult to find clear trends. For instance, the W03 and GAL09 sets give very similar results for Model I, but not as similar as for Models II and III.

If we discard the S01 set (which is based on an unpublished preliminary analysis), we can conclude that the SystUnc on the transport parameters related to the choice of the production cross-section is $\lesssim 20 \%$. This figure is similar to the uncertainty quoted for the parameterisation of the cross-section themselves $(\sim 10-20 \%)$.

\subsection{Influence of a systematic energy bias in W03 cross-sections}

A striking result of the updated cross-section formulation of W03 (Webber et al. 2003) compared to WKS98 (Webber et al. 1998) was a systematic energy bias (smaller cross-sections at

\footnotetext{
${ }^{1}$ http://galprop.stanford.edu/web_galprop/galprop_ home.html
}

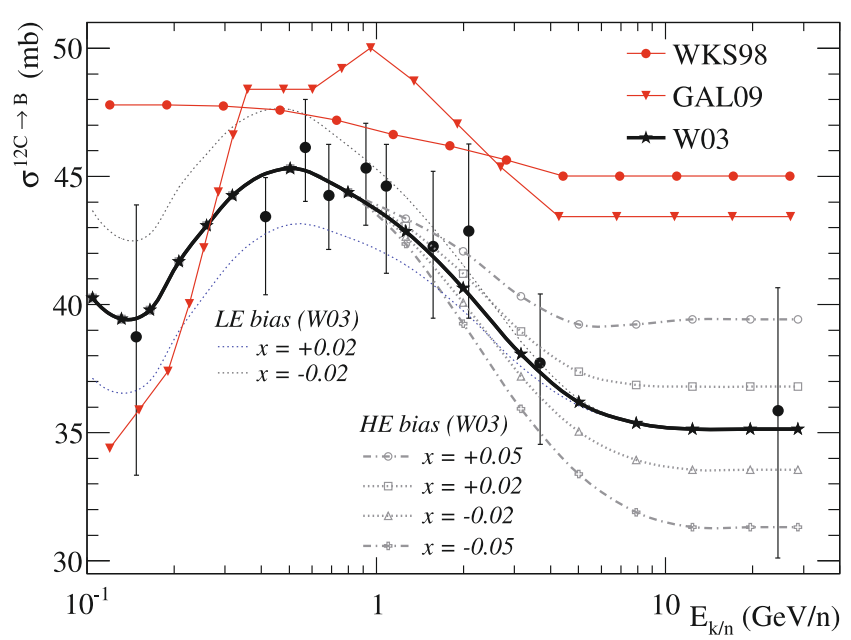

Fig. 3. Production cross-section for ${ }^{12} \mathrm{C}+\mathrm{H} \rightarrow{ }^{10,11} \mathrm{~B}$ (adapted from Webber et al. 2003). The standard sets are shown as solid lines (WKS98: red dots; GAL09: red down triangles; W03: black stars), and the biased sets in dotted $(|x|=0.02)$ and dashed $(|x|=0.05)$ lines.

higher energy). To address the effect of a possible residual bias, we allow for a systematic energy bias in all production crosssections, either at low energy (LE) or at high energy (HE). Biased sets are obtained from the reference set W03 by applying a factor $\left(E_{\mathrm{k} / \mathrm{n}} / 10\right)^{-x}$ below $10 \mathrm{GeV} / \mathrm{n}$ (LE bias), and $\left(E_{\mathrm{k} / \mathrm{n}}\right)^{x}$ above $1 \mathrm{GeV} / \mathrm{n}$ (HE bias, but left constant above $10 \mathrm{GeV} / \mathrm{n}$ ). This is illustrated for the ${ }^{12} \mathrm{C} \rightarrow\left({ }^{10} \mathrm{~B}+{ }^{11} \mathrm{~B}\right)$ cross-section, as shown in Fig. 3. As seen from this figure and the experimental data, biases $x$ as large as 0.05 are marginally consistent with the data at low energy, but are acceptable at high energy.

In Table 8, we only report the results of the high-energy biases on the best-fit values of the transport parameters. We checked that the result of a low-energy or a high-energy bias gives the same trends. Quite naturally, there is a significant correlation of the bias with the transport parameters. We note that if $\delta$ increases, all the other transport parameters $K_{0}, V_{\mathrm{a}}$, and $V_{\mathrm{c}}$ decrease. In principle, we would expect that $\delta$ increases if the bias is positive: larger cross-sections at high-energy produce more secondaries at high-energy, requiring a larger $\delta$ to match the same $\mathrm{B} / \mathrm{C}$ data. This is observed for Models 0 and II. However, the reverse effect is observed for Models I and III, i.e., for models with convective wind.

Such an exercise has limitations since it is not realistic to expect such systematic energy biases for all production channels. However, it gives some trend and further confirms that Model III cannot be reconcile with small $\delta$.

\subsection{Summary for the influence of fragmentation cross-sections}

The results when using different production cross-section sets are difficult to interpret. To check that the observed trends do not come from an error in the code, we compared our results with the LBM analysis of Webber et al. (2003). These authors find a significantly smaller escape length dependence on rigidity (from $P^{0.6}$ to $\left.P^{0.5}\right)$, going from the WKS98 to the W03 set. From both an LBM analysis with our propagation code and a DM analysis using the LBI case (see Table 5) with the addition of a rigidity cut-off (not discussed in this paper), we also find this decrease of $\Delta \delta=0.1$. This is also confirmed for the more standard Model 0 (pure diffusion) where we go from 0.53 to 0.40 . 

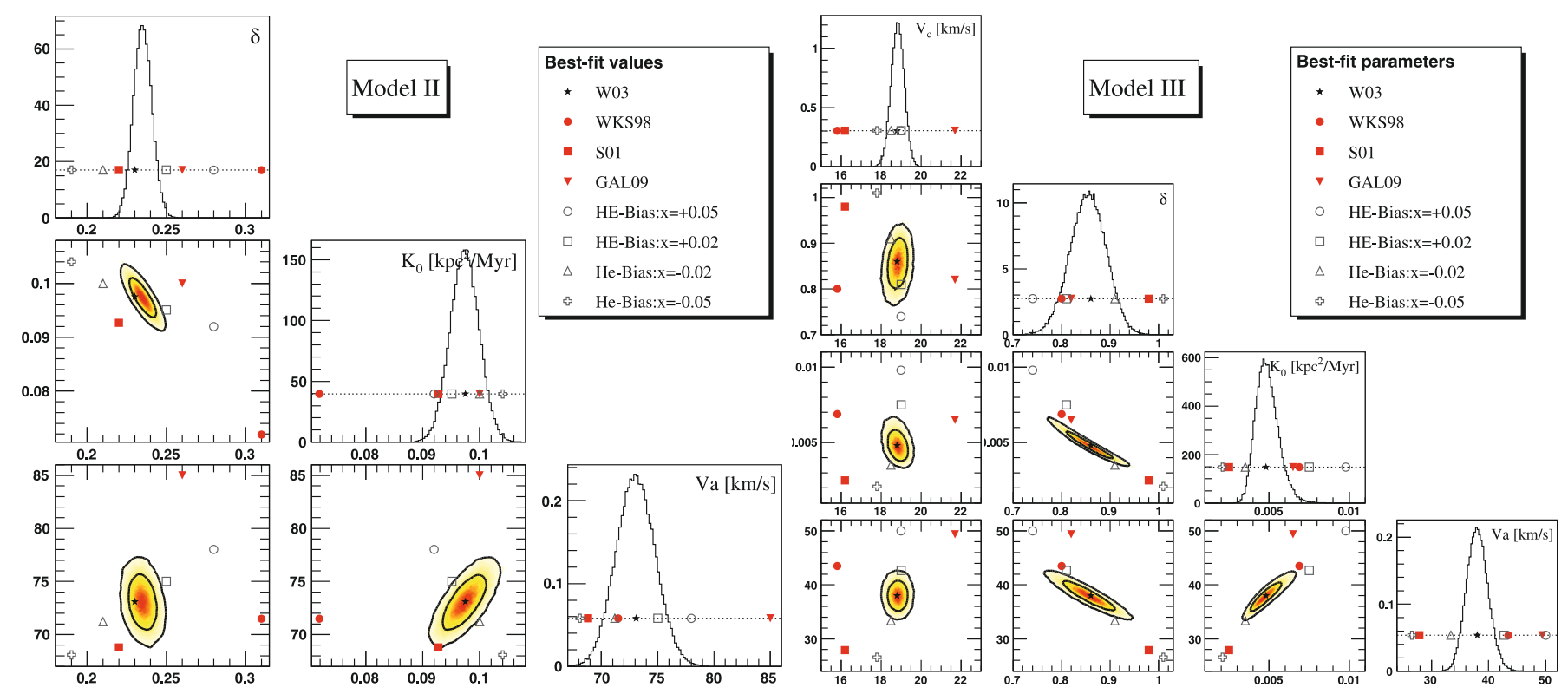

Fig. 4. Left panel: Model II (pure reacceleration). Right panel: Model III (reacceleration and convection). PDF of the transport parameters (for the reference inputs) as obtained in Paper II, along with the best-fit values for different production cross-section sets.

Table 8. High-energy biases on the W03 cross-section set.

\begin{tabular}{cccccc}
\hline \hline $\begin{array}{c}\text { W03 } \\
\text { HE bias } x\end{array}$ & $\begin{array}{c}K_{0}^{\text {best }} \times 10^{2} \\
\left(\mathrm{kpc}^{2} \mathrm{Myr}^{-1}\right)\end{array}$ & $\delta^{\text {best }}$ & $\begin{array}{c}V_{\mathrm{c}}^{\text {best }} \\
\left(\mathrm{km} \mathrm{s}^{-1}\right)\end{array}$ & $\begin{array}{c}V_{\mathrm{a}}^{\text {best }}\left(\mathrm{km} \mathrm{s}^{-1}\right) \\
\chi^{2} / \text { d.o.f. }\end{array}$ \\
\hline 0: +0.05 & 4.05 & 0.45 & $\ldots$ & $\ldots$ & 26.5 \\
0: +0.02 & 4.06 & 0.42 & $\ldots$ & $\ldots$ & 27.7 \\
0: +0.00 & 4.08 & 0.40 & $\ldots$ & $\ldots$ & 28.8 \\
0: -0.02 & 4.09 & 0.38 & $\ldots$ & $\ldots$ & 30.1 \\
0: -0.05 & 4.13 & 0.34 & $\ldots$ & $\ldots$ & 32.5 \\
I: +0.05 & 0.61 & 0.89 & 13.7 & $\ldots$ & 12.1 \\
I: +0.02 & 0.49 & 0.91 & 13.6 & $\ldots$ & 11.4 \\
I: +0.00 & 0.42 & 0.93 & 13.5 & $\ldots$ & 11.2 \\
I: -0.02 & 0.35 & 0.95 & 13.4 & $\ldots$ & 11.2 \\
I: -0.05 & 0.27 & 0.98 & 13.1 & $\ldots$ & 11.8 \\
II: +0.05 & 9.20 & 0.28 & $\ldots$ & 78.0 & 3.26 \\
II: +0.02 & 9.51 & 0.25 & $\ldots$ & 75.0 & 4.13 \\
II: +0.00 & 9.76 & 0.23 & $\ldots$ & 73.1 & 4.73 \\
II: -0.02 & 10.0 & 0.21 & $\ldots$ & 71.2 & 5.35 \\
II: -0.05 & 10.4 & 0.19 & $\ldots$ & 68.1 & 6.27 \\
III: +0.05 & 0.98 & 0.74 & 19.0 & 50.0 & 1.01 \\
III: +0.02 & 0.75 & 0.81 & 19.0 & 42.7 & 1.22 \\
III: +0.00 & 0.48 & 0.86 & 18.8 & 38.0 & 1.47 \\
III: -0.02 & 0.35 & 0.91 & 18.5 & 33.4 & 1.88 \\
III: -0.05 & 0.21 & 1.01 & 17.8 & 26.6 & 2.94 \\
\hline
\end{tabular}

Notes. Models 0 , I, II, and III for $L=4 \mathrm{kpc}$. The various sets are obtained after multiplying the W03 set by $\left(E_{\mathrm{k} / \mathrm{n}}\right)^{x}$ above $1 \mathrm{GeV} / \mathrm{n}$ (and kept constant above $10 \mathrm{GeV} / \mathrm{n}$ ), with $x=+0.05,+0.02,0,-0.02,-0.05$. (corresponding to bottom to top curves on the right-hand side of Fig. 3).

We thus have to conclude, as seen from Table 7 , that the effect of these cross-section sets is not the same for different classes of models: it strongly depends on the presence or absence of convection and, to a lesser extent, on reacceleration. Nevertheless, for all models, the typical scatter in the best-fit values is a factor of 2 for $K_{0}, \sim 50 \%$ for $V_{\mathrm{a}}, \sim 10 \%$ for $\delta$, and $\sim 5 \%$ for

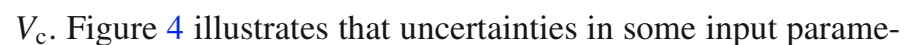
ters (here the production cross-sections) provide larger SystUnc on the transport parameters than their StatUnc calculated using the MCMC technique (taken from Paper II).

As for the other effects inspected in previous sections, Model III always provide $\delta$ larger than $\sim 0.6$. Model II provides values of $\delta$ in the correct range for a Kolmogorov spectrum, but it never does better than Model III in terms of the goodness-of-fit.

\section{Influence of an energy bias in HEAO-3 B/C data}

It is obvious that most of the results derived in this paper rely on the confidence we place in the HEAO-3 data, since they are the most constraining data to date. Their very small error bars at high energy could over-constrain $\delta$. Several other studies use large error bars for high energy $\mathrm{B} / \mathrm{C} \mathrm{HEAO}-3$ points, contradicting the prescription given in the original paper (Engelmann et al. 1990), and this could bias their results. As an exercise, we allowed for an energy bias in these data. This approach is disputable so we do not wish to defend it strongly. We inspected whether a small bias in the data strongly affects the best-fit values of the transport parameters.

The HEAO-3 data are biased above $E_{\mathrm{c}}$, using $\left(E_{\mathrm{k} / \mathrm{n}} / E_{\mathrm{c}}\right)^{x}$ for $E_{\mathrm{k} / \mathrm{n}}>E_{\mathrm{c}}$, with $E_{\mathrm{c}}=2.9 \mathrm{GeV} / \mathrm{n}$, as shown in Fig. 5. As in previous sections, we fit Models 0 , I, II, and III on dataset F (as shown in Fig. 5), where the HEAO-3 data have been replaced by the biased ones. The results have been gathered in Table 9. Intuitively, in a similar fashion as for the bias in the cross-sections, we expect that a higher value of the $\mathrm{B} / \mathrm{C}$ data at high energy (positive bias) leads to a smaller $\delta$. This is what is observed for all models. The effect of the bias on $\delta$ is at its maximum for the pure diffusion model (Model 0 ), where $\Delta \delta \sim 0.2$. For Models II and III, the effect is less pronounced owing to the presence of reacceleration, with a change of only $\Delta \delta \sim 0.1$. However, Model III (convection and reacceleration), which is statistically preferred over Model II, still points to uncomfortably high values for $\delta$. The only way out would be to have a non-systematic energy effect in the data. For instance, the result on the fit to the recently 


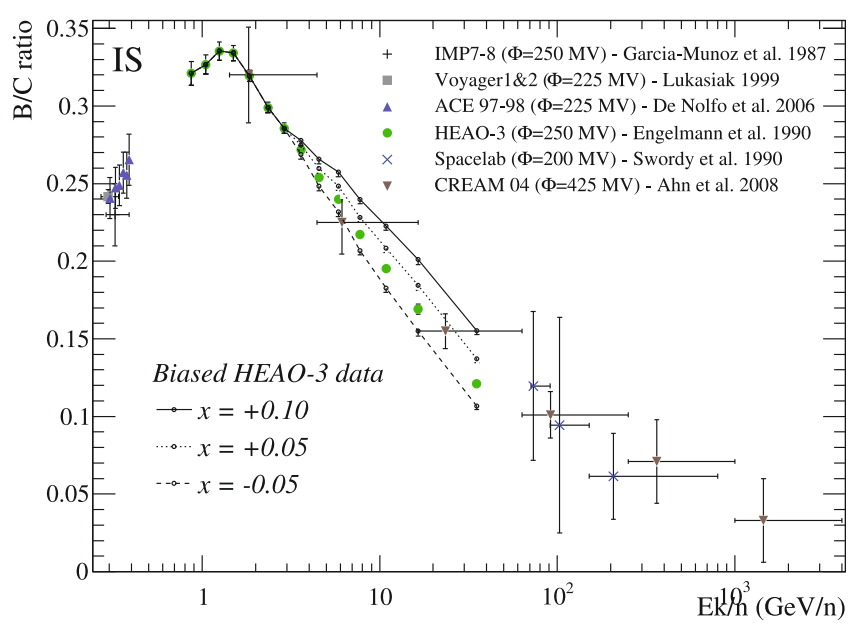

Fig. 5. Demodulated $\mathrm{B} / \mathrm{C}$ data used in this paper (denoted dataset $\mathrm{F}$ ). Biased HEAO-3 data are shown as solid, dashed, and dotted lines.

Table 9. Influence of biasing HEAO-3 data.

\begin{tabular}{cccccc}
\hline \hline $\begin{array}{c}\text { HEAO-3 } \\
\text { HE bias } x\end{array}$ & $\begin{array}{c}K_{0}^{\text {best }} \times 10^{2} \\
\left(\mathrm{kpc}^{2} \mathrm{Myr}^{-1}\right)\end{array}$ & $\delta^{\text {best }}$ & $\begin{array}{c}V_{\mathrm{c}}^{\text {best }} \\
\left(\mathrm{km} \mathrm{s}^{-1}\right)\end{array}$ & $\begin{array}{c}V_{\mathrm{a}}^{\text {best }} \\
\left(\mathrm{km} \mathrm{s}^{-1}\right)\end{array}$ & $\chi^{2} /$ d.o.f. \\
\hline $0:+0.10$ & 4.97 & 0.28 & $\ldots$ & $\ldots$ & 22.5 \\
$0:+0.05$ & 4.50 & 0.34 & $\ldots$ & $\ldots$ & 25.2 \\
O: +0.00 & 4.08 & 0.40 & $\ldots$ & $\ldots$ & 28.8 \\
0: -0.05 & 3.69 & 0.46 & $\ldots$ & $\ldots$ & 33.0 \\
I: +0.10 & 0.45 & 0.81 & 13.4 & $\ldots$ & 9.77 \\
I: +0.05 & 0.44 & 0.87 & 13.5 & $\ldots$ & 10.2 \\
I: +0.00 & 0.42 & 0.93 & 13.5 & $\ldots$ & 11.2 \\
I: -0.05 & 0.40 & 0.99 & 13.6 & $\ldots$ & 12.5 \\
II: +0.10 & 10.1 & 0.17 & $\ldots$ & 56.6 & 4.54 \\
II: +0.05 & 9.95 & 0.20 & $\ldots$ & 64.8 & 4.27 \\
II: +0.00 & 9.76 & 0.23 & $\ldots$ & 73.1 & 4.73 \\
II: -0.05 & 9.56 & 0.27 & $\ldots$ & 81.5 & 5.79 \\
III: +0.10 & 0.31 & 0.85 & 19.1 & 30.0 & 1.32 \\
III: +0.05 & 0.42 & 0.83 & 19.0 & 34.8 & 1.16 \\
III: +0.00 & 0.48 & 0.86 & 18.8 & 38.0 & 1.47 \\
III: -0.05 & 0.50 & 0.90 & 18.7 & 40.0 & 2.18 \\
\hline
\end{tabular}

Notes. Models 0, I, II, and III for $L=4 \mathrm{kpc}$, where HEAO-3 data are biased using the formula $\left(E_{\mathrm{k} / \mathrm{n}} / E_{\mathrm{c}}\right)^{x}$ for $E_{\mathrm{k} / \mathrm{n}}>E_{\mathrm{c}}$, with $E_{\mathrm{c}}=2.9 \mathrm{GeV} / \mathrm{n}$ and $x=+0.1,+0.05,0 .,-0.05$.

published AMS-01 data (Tomassetti \& AMS-01 Collaboration 2009) leads to $\delta \approx 0.5$ (see Paper II).

Finally, for completeness, we checked that the effect of the solar modulation parameters (we took $\Delta \phi \pm 50 \mathrm{MV}$ for the HEAO-3 data) was negligible on all the derived transport parameters.

\section{Summary and discussion}

The guiding questions for this paper were the following. First, do uncertainties in the input ingredients lead to systematic uncertainties (SystUnc) on the derived transport parameters greater than their statistical uncertainties (StatUnc)? Second, can we reproduce the various values of $\delta$ given in the literature, and which one should be preferred? We elaborate on the answers below before concluding.

\subsection{SystUnc and StatUnc: which ones dominate?}

From the analysis of Paper II, the StatUnc found for the various transport parameters typically fall in the range 5-10\% (see Fig. 4). The SystUnc generated by each input we varied are summarised below.

- A change of $x \%$ in the gas surface density $\Sigma_{\text {ISM }}$ translates into a change of $x \%$ for all transport parameters but $\delta$, which is unaffected. Such a simple scaling can be used for comparing models in the literature, as different $\Sigma_{\text {ISM }}$ are generally used. Depending on how confident we are in the measurement of $\Sigma_{\text {ISM }}$, we may conclude that, for this input, the generated SystUnc is similar to or larger than the StatUnc.

- The transport parameters are very sensitive to the low-energy spectral shape of the source parameter $\eta_{\mathrm{S}}$, but not to the source slope $\alpha$. Both parameters can be determined by including data of a primary flux in the fit. When doing so, the SystUnc and StatUnc are of the same order.

- If we let $\eta_{\mathrm{T}}$ - which parameterises the low-energy shape of the diffusion coefficient - free in the range $[-2 ., 1$.$] , the$ SystUnc completely dominate the StatUnc. Diffusion coefficients with a sharp turn-off at low-energy are possible, as shown in Fig. 1 of Ptuskin et al. (2006) (their dashed line corresponds to our $\eta_{\mathrm{T}}=-2$ ).

- For the cross sections, as seen in Fig. 4, the SystUnc can also be larger than the StatUnc for some of the transport coefficients. The use of other secondary-to-primary ratios, such as $\mathrm{Li} / \mathrm{C}$ and $\mathrm{Be} / \mathrm{C}$, could help to cross-check the consistency of each cross-section set, and thus decrease the SystUnc.

These conclusions hold for all classes of models ( 0 , I, II, and III).

\subsection{Which value of $\delta$ should we trust?}

The two key ingredients for determinaning $\delta$ are i) the lowenergy spectral shape of the diffusion coefficient as parameterised by $\eta_{\mathrm{T}}$ [see Eq. (10)], and ii) the presence or absence of a wind. These two effects allow $\delta$ to reach values as low as 0.2 up to 0.9. Such a wide range is also found in Jones et al. (2001), depending on the model they consider (convection, turbulent diffusion, stochastic reacceleration).

We recover $\delta \approx 0.3$ in the pure reacceleration case (no convection), as in other propagation codes (Strong \& Moskalenko 1998; Jones et al. 2001). As soon as a convective wind is included, the diffusion slope is large $(\delta \approx 0.8)$, as obtained in other studies (Jones et al. 2001; Maurin et al. 2001). The effect of the low-energy shape of the diffusion coefficient is illustrated by the fact that standard LBM and standard diffusion models lead to different $\delta$ (more details are given in Putze, Derome \& Maurin, ICRC 2009). For LBM, which are equivalent to diffusion models if $K(E)=K_{0} \mathcal{R}^{\delta}$, we recover $\delta \approx 0.5-0.6$ (Webber et al. 2003, Paper I.

It is reassuring to see that the results of the various propagation codes used in the literature are consistent. However, this does not settle the question of which value of $\delta$ we should trust. In principle, one advantage of our analysis is that it should clearly point to a preferred value for $\delta$, by a mere comparison of the best $\chi^{2}$ values. Indeed, in almost all settings considered, Model III (convection and reacceleration) performs better than 


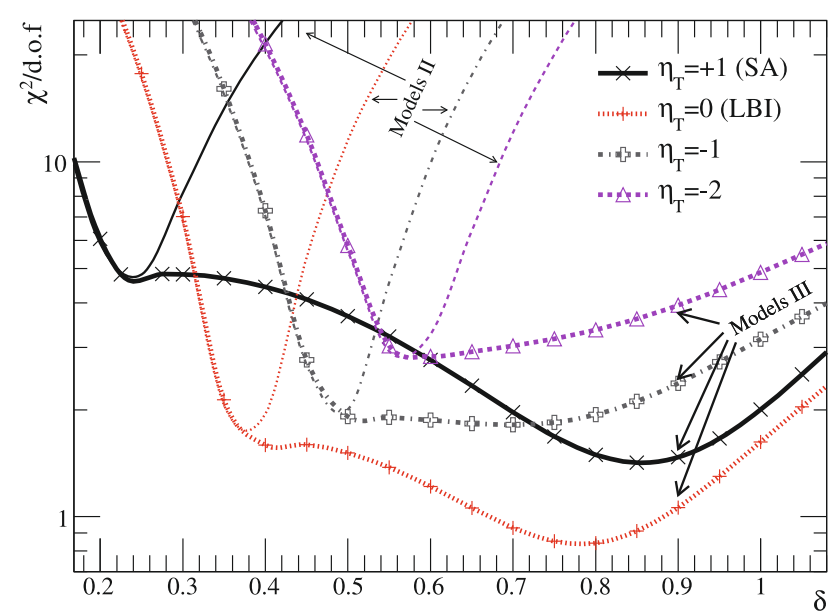

Fig. 6. Evolution of $\chi_{\min }^{2} /$ d.o.f. as a function of $\delta$ for III (thick lines with symbols) and II (thin lines). The four cases correspond to different $\eta_{\mathrm{T}}$ (low-energy shape of the diffusion coefficient).

any other, the second best being Model II (no convection). This behaviour is illustrated by the $\chi_{\min }^{2}$ dependence as a function of $\delta$, as shown in Fig. 6. For a standard diffusion coefficient (black-solid curve), there is a clear minimum at high $\delta$, and a less marked local minimum at low $\delta$. This second minimum matches the $\chi_{\min }^{2}$ of Model II (black-dashed curve). The value of the $\chi_{\min }^{2}$ slightly decreases when $\eta_{\mathrm{T}}$ decreases (red curves), then increases for $\eta_{\mathrm{T}} \lesssim 0$ (grey and violet curves). In the process, the best-fit value for $\delta$ is displaced to smaller values, the local minimum at low $\delta$ overtaking and merging with the high $\delta$ one.

For standard diffusion schemes (SA), the best class of models is Model III. The main problem is that whatever ingredients are changed in this model, we are always left with $\delta \gtrsim 0.6$, and most of the time with even higher values, $\sim 0.9$. Such high values, always associated to the presence of the constant convective wind, are difficult to explain. Should we choose to discard this Model III, we would be left with the task of explaining why the statistical analysis fails in the context of CR physics. A way out is provided, as seen in Fig. 6, if we let free $\eta_{\mathrm{T}}$ (low-energy shape of the diffusion coefficient). Indeed, the only case where Model II (reacceleration only) performs as Model III is for $\eta_{\mathrm{T}}$ in the range $-3 \lesssim \eta_{\mathrm{T}} \lesssim-1.5$. In such a configuration, the bestfit value for the convective velocity of Model III drops to zero, so that the best model only has reacceleration (i.e. equivalent to Model II): we term this class of models III/II. The next best-fit class of models is obtained if we discard reacceleration (Model Ilike) and again let $\eta_{\mathrm{T}}$ go free. As for the previous case, the bestfit value for the convective velocity drops to zero: we denote this class $\mathrm{I} / 0$. In terms of the best $\chi_{\min }^{2}$, the standard diffusion Model II has only the fourth-best $\chi^{2}$ value. The best-fit values for these four classes of models are gathered in Table 10, sorted according to their figure of merit.

The best-fit curves for these four relevant configurations are shown in Fig. 7. From this graphic view, we can see that Model II matches the data best at very-low energy, Model III at intermediate energies, and Models $\mathrm{O} / \mathrm{I}$ and III/II at high energy. While we found that a systematic bias in HEAO-3 data would not change the ordering of which is the best model (see Sect. 8), we see from Fig. 7 that a more complicated pattern in the data may be more effective in changing which model is best.

To summarise, when a standard scheme for the diffusion coefficient is assumed, Model III is always preferred over Model II.
Table 10. Best-fit parameters for a few selected configurations.

\begin{tabular}{lcccccc}
\hline \hline Model & $\eta_{\mathrm{T}}$ & $\begin{array}{c}K_{0} \times 10^{2} \\
\left(\mathrm{kpc}^{2} \mathrm{Myr}^{-1}\right)\end{array}$ & $\delta$ & $\begin{array}{c}V_{\mathrm{c}} \\
\left(\mathrm{km} \mathrm{s}^{-1}\right)\end{array}$ & $\begin{array}{c}V_{\mathrm{a}} \\
\left(\mathrm{km} \mathrm{s}^{-1}\right)\end{array}$ & $\chi^{2} /$ d.o.f. \\
\hline $\mathrm{III}^{\dagger}$ & $\mathrm{SA}$ & 0.481 & 0.856 & 18.84 & 37.98 & 1.47 \\
$\mathrm{III} / \mathrm{II}^{\star}$ & -1.3 & 3.161 & 0.512 & 0. & 45.35 & 2.26 \\
$\mathrm{I} / 0^{\ddagger}$ & -2.61 & 2.054 & 0.613 & 0. & $\ldots$ & 3.29 \\
$\mathrm{II}^{\dagger}$ & $\mathrm{SA}$ & 9.753 & 0.234 & $\ldots$ & 73.14 & 4.73 \\
\hline
\end{tabular}

Notes. Standard models refer to SA diffusion coefficients (see Table 5). Alternative models show their different values for $\eta_{\mathrm{T}}$, as parameterised from Eq. (10). ${ }^{(\dagger)}$ Best-fit transport parameters for standard Model II and III. ${ }^{(\grave{)})}$ Best-fit parameters with $\eta_{\mathrm{T}}$ free (no reacceleration). ${ }^{(\star)}$ Bestfit parameters for fixed $\eta_{\mathrm{T}}$.

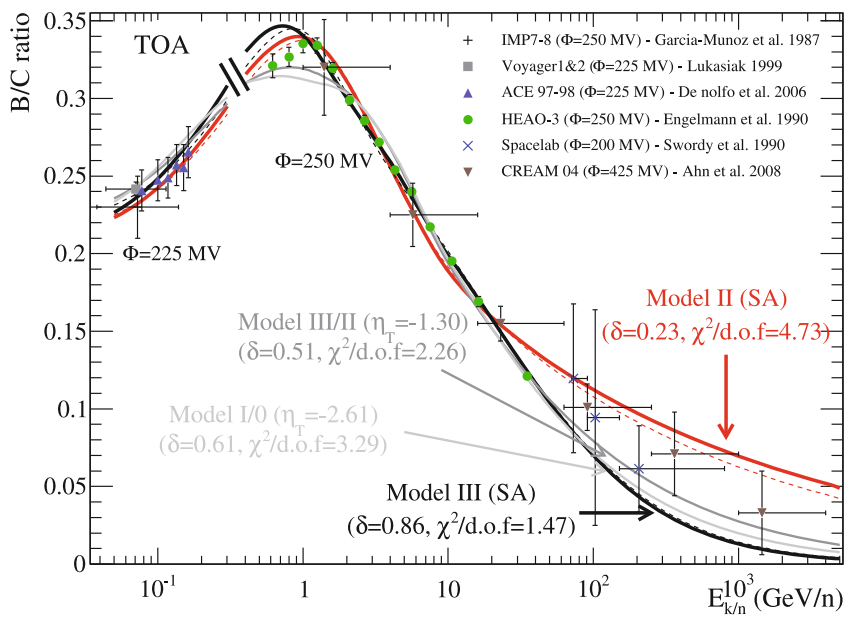

Fig. 7. Best-fit B/C ratio for standard Model II (thick red) and III (thick black) using the reference setting (solid and thin dashed lines are for the cross-section sets W03 and GAL09). The special best-fit Models $\mathrm{O} / \mathrm{I}$ and III/II are also plotted in thin grey lines (light and dark shade respectively).

However, the latter seems to agree more with theoretical expectations for $\delta$. Model II is also more consistent with the measured propagated slope $\gamma=\alpha+\delta$. Because of this oddity, we may not be able to give a definite answer about the value of $\delta$. This is further complicated when $\eta_{\mathrm{T}}$ is left free; in that case, best models are always without convection, and even the pure diffusive model is redeemed (although both have difficulty reproducing the $\mathrm{B} / \mathrm{C}$ peak at $\mathrm{GeV} / \mathrm{n}$ energies). We see that many uncertainties show up at $\mathrm{GeV} / \mathrm{n}$ energies. As also illustrated by the various predictions for various $\delta$ in Fig. 7, the higher the energy, the closer we can expect to reach the purely diffusive regime. As a result, high-energy $\mathrm{B} / \mathrm{C}$ data are desired to unambiguously pinpoint the value of $\delta$.

\subsection{Conclusion}

In the past years, we have promoted and used a model favouring both convection and reacceleration (e.g., Maurin et al. 2001, 2002, and subsequent studies) from statistical criteria. The main and known problem of this model lies in its uncomfortably high value for $\delta(\delta \sim 0.8)$. Such a model is also preferred in the present study. In addition, we found that the high value for $\delta$ is extremely resilient to any change in the setting, which leaves us with several alternatives: assume that there are complicated biases in the data that conspire to give high $\delta$ in such models, 
that this high value of $\delta$ is real (in that case it needs to be explained theoretically), or that any model with convection should be excluded (which contradicts the fact that winds are observed in many galaxies). Even if we adopt the last alternative, no firm conclusions can be drawn on the value of $\delta$. Indeed, if the statistical analysis is relaxed, a large category of models are redeemed, attaining any value for $\delta$ between 0.3 and 0.9 . These models may be purely diffusive, with convection and/or reacceleration, and are very sensitive to the shape of the low-energy diffusion coefficient (which is not prescribed theoretically for the moment). Data at higher energy are needed to solve this question. More constraints can also be obtained by combining several secondary-to-primary ratios (e.g., Webber 1997a,b). This is left for a later study.

This study has limitations. For instance, we only varied the source and diffusion parameters according to simple parameterisations. More complicated dependences could have been inspected. However, it is worth recalling that it may be dangerous to introduce too many ad hoc prescriptions, because the statistical meaning - already unclear when comparing the different classes of models - becomes less and less obvious as the number of parameters and models tested increase. In the framework of homogeneous and isotropic diffusion coefficients, a maybe more important issue is the question of the Galactic wind. A constant wind was chosen because of the simplicity of the solutions (of the corresponding diffusion equation). On the one hand, Galactic winds are ubiquitous. On the other, as shown in this study, constant wind cannot accommodate a realistic slope of the diffusion coefficient. A linear wind may provide different results. We are implementing a numerical solver in our propagation code to inspect this issue. Another possibility that cannot be ruled out is that the HEAO-3 data suffer from non-trivial systematics.

Finally, the starting point of the paper was a comparison between systematic uncertainties (generated by uncertainties in the input ingredients) and statistical uncertainties for the values of the transport parameters. That the former can be larger than the latter shows that many efforts are still needed in CR physics, especially for the production cross-sections, before one can take full advantage of any statistical analysis, as performed in Papers I and II with an MCMC technique. Some of these issues may be resolved as new data on cosmic-ray nuclei are being released (CREAM, PAMELA, TRACER).

Acknowledgements. We thank W. R. Webber for providing us with the nuclear production cross-sections discussed in Webber et al. (2003) and for useful suggestions. We also thank C. Combet for a careful reading of the paper. A.P. is grateful for financial support from the Swedish Research Council (VR) through the Oskar Klein Centre. We acknowledge the support of the French ANR (grant ANR-06-CREAM).

\section{References}

Ahn, H. S., Allison, P. S., Bagliesi, M. G., et al. 2008, Astropart. Phys., 30, 133 Ave, M., Boyle, P. J., Gahbauer, F., et al. 2008, ApJ, 678, 262

de Nolfo, G. A., Moskalenko, I. V., Binns, W. R., et al. 2006, Adv. Space Res., 38,1558

Donato, F., Maurin, D., Brun, P., Delahaye, T., \& Salati, P. 2009, Phys. Rev. Lett., 102,071301

Engelmann, J. J., Ferrando, P., Soutoul, A., Goret, P., \& Juliusson, E. 1990, A\&A, 233, 96

Garcia-Munoz, M., Simpson, J. A., Guzik, T. G., Wefel, J. P., \& Margolis, S. H. 1987, ApJS, 64, 269

Jones, F. C., Lukasiak, A., Ptuskin, V., \& Webber, W. 2001, ApJ, 547, 264

Lionetto, A. M., Morselli, A., \& Zdravkovic, V. 2005, JCAP, 9, 10

Lukasiak, A., McDonald, F. B., \& Webber, W. R. 1999, 3, 41

Maurin, D., Donato, F., Taillet, R., \& Salati, P. 2001, ApJ, 555, 585

Maurin, D., Taillet, R., \& Donato, F. 2002, A\&A, 394, 1039

Maurin, D., Taillet, R., \& Combet, C. 2006, [arXiv:astro-ph/0612714]

Osborne, J. L., \& Ptuskin, V. S. 1988, SvA Lett., 14, 132

Ptuskin, V. S., Moskalenko, I. V., Jones, F. C., Strong, A. W., \& Zirakashvili, V. N. 2006, ApJ, 642, 902

Putze, A., Derome, L., Maurin, D., Perotto, L., \& Taillet, R. 2009, A\&A, 497, 991 (Paper I)

Putze, A., Derome, L., \& Maurin, D. 2010, A\&A, 516, A66 (Paper II)

Schlickeiser, R. 2002, Cosmic Ray Astrophysics, ed. R. Schlickeiser

Seo, E. S., \& Ptuskin, V. S. 1994, ApJ, 431, 705

Strong, A. W., \& Moskalenko, I. V. 1998, ApJ, 509, 212

Swordy, S. P., Mueller, D., Meyer, P., L'Heureux, J., \& Grunsfeld, J. M. 1990, ApJ, 349, 625

Taillet, R., \& Maurin, D. 2003, A\&A, 402, 971

Tomassetti, N., \& AMS-01 Collaboration. 2009, in International Cosmic Ray Conference, 1

Webber, W. R. 1997a, Adv. Space Res., 19, 755

Webber, W. R. 1997b, Space Sci. Rev., 81, 107

Webber, W. R., Kish, J. C., Rockstroh, J. M., et al. 1998, ApJ, 508, 940

Webber, W. R., Kish, J. C., \& Schrier, D. A. 1990, Phys. Rev. C, 41, 566

Webber, W. R., Soutoul, A., Kish, J. C., \& Rockstroh, J. M. 2003, ApJS, 144, 153 